\title{
1D numerical study on hydrogen injection enabling ultra-lean combustion in a small gasoline Spark Ignition engine
}

\author{
Luigi Teodosio ${ }^{1, *}$, Dino Pirrello ${ }^{2}$, and Luca Marchitto ${ }^{1}$ \\ ${ }^{1}$ Istituto Motori CNR, Naples, Italy \\ ${ }^{2}$ Independent Researcher, Catania, Italy
}

\begin{abstract}
This paper deals with the effects of hydrogen port injection on combustion evolution, efficiency and exhaust emissions of a small turbocharged gasoline Spark-Ignition engine through a 1D numerical code. First, the experiments on the base engine architecture are performed at different speeds and at low/medium loads. The experimental findings are used to validate a $1 \mathrm{D}$ model of the whole engine, developed within a commercial code. 1D model is also refined with "user-defined" sub-models for an accurate description of the in-cylinder phenomena, namely turbulence, combustion, heat transfer, and emissions. In a second step, 1D model is virtually modified through the installation of an hydrogen injector in each intake runner, while the combustion sub-model also accounts for the impact of hydrogen addition on the laminar flame speed through a dedicated correlation. 1D simulations are performed at low/medium loads and fixed speed of $2250 \mathrm{rpm}$ with $5 \%$ of hydrogen by volume in the intake air. Numerical investigations show that hydrogen addition to gasoline/air mixtures allows relevant efficiency benefits (up to a maximum percent gain of 19\%), while the NO emissions are almost eliminated. Consequently, hydrogen-boosted combustion represents a potential solution to achieve very high efficiency and reduced pollutant emissions of gasoline spark ignition engines equipped with a conventional combustion system.
\end{abstract}

\section{Introduction}

In the last years the automotive industry is addressing many efforts towards the development of clean and efficient Spark Ignition (SI) internal combustion engines (ICEs) in order to comply with the stringent pollutant and $\mathrm{CO}_{2}$ emission legislations for vehicle homologation [1]. To this aim, various techniques are being successfully implemented in SI engines, involving different levels of architecture complexity, costs, reliability and performance improvements.

Common technical solutions for SI ICEs include the downsizing coupled to turbocharging [2], innovative valve strategies [3], increased or variable compression ratio

\footnotetext{
* Corresponding author: 1.teodosio@im.cnr.it
} 
(CR) $[4,5,6]$, external cooled exhaust gas recirculation (EGR) $[7,8]$, water injection $[9$, $10]$, employment of alternative fuels $[11,12]$, and pre-chamber ignition devices [13]. These solutions allow some benefits in the engine performance but they highly depend on the investigated operating conditions $[14,15]$. In addition to the above recalled technologies, an increasingly attention is being focused to the alternative fuels to be blended with commercial gasoline in order to enable stable lean combustion. Hydrogen is proved to be a good fuel for vehicles when used as a combustion enhancer in gasoline SI engines [16]. Indeed, the physical and chemical properties of hydrogen allow to support the flame development and to extend the dilution limits with improved combustion stability [17]. The strategy to use a small amount of hydrogen as an additive to a gasoline SI engine can lead to relevant advantages in terms of engine thermal efficiency, pollutant emissions and also contributing to reduce the cycle-by-cycle variation [18].

The current literature reports various papers regarding the experimental influence of hydrogen injection on the performance, combustion and emissions of gasoline spark ignition engines. For example, Ji et al. [16] performed measurements to analyze the influence of hydrogen addition on combustion and emission of a gasoline spark ignition engine. They showed that hydrogen blending leads to increased flame development, improved brake thermal efficiency (percent gain of about $27 \%$ with $3 \%$ vol. $\mathrm{H}_{2}$ at $\lambda=2$ ), reduced emissions and cycle-by-cycle variation limited at $10 \% \mathrm{COV}_{\text {IMEP. D'Andrea [17] }}$ also studied the impact of hydrogen injection in the intake sub-system on the combustion evolution of a modified carburetor engine, confirming the hydrogen-related increase in the combustion speed. Tahtough et al. [19] investigated a single-cylinder engine demonstrating that the combination of hydrogen supplementation with lean operation positively affects the exhaust emissions. On the other hand, very few articles deal with the numerical study of hydrogen addition effects on SI engine behaviour. Iafrate et al. [18] carried out 3D CFD calculations at lean condition ( $\lambda=2$ ) with $3 \%$ hydrogen by volume considering both low and medium/high loads. They also developed a kinetical-derived laminar flame speed (LFS) correlation and a tabulated kinetics of ignition (TKI) model implemented into the 3D code. This study demonstrated that hydrogen enhances the combustion speed, the knock resistance and also improves the indicated efficiency up to about $48 \%$ under knock-limited operation.

In the present paper, the influence of hydrogen injection on the efficiency, combustion and exhaust emissions of a small gasoline Spark Ignition engine is investigated by a 1D numerical approach. First, engine characteristics and the related experimental activity are described. A 1D model of the entire engine is developed in a commercial code and validated against a proper set of experimental findings at low/medium loads, both in terms of overall performance, combustion evolution, $\mathrm{NO}$ and $\mathrm{CO}$ emissions.

In a second stage, 1D engine model is applied, in a predictive way, to explore the potentials of hydrogen injection in the intake sub-system with the aim to promote an ultralean combustion. The analyses are limited to a fixed speed of $2250 \mathrm{rpm}$ and at knock-free operations. A first investigation is performed in a single operating point $(2250 \mathrm{rpm}$ and 3 bar IMEP, labelled as 2250@3) under hydrogen injection mode, where a sweep of relative air/fuel ratio $(\lambda)$ is realized up to a maximum level of 2 in order to identify the trends of the relevant engine performance and the related maximum acceptable advantages. A second analysis is carried out at various loads and at constant speed of $2250 \mathrm{rpm}$ to compare the results of engine stoichiometric operation with those resulting from the ultra-lean mixture operation where the combustion is boosted with $5 \%$ of hydrogen by volume in the intake air. The latter analysis reveals significant indicated efficiency and emission benefits under hydrogen injection. The maximum computed gain for the indicated efficiency is equal to about 19\%, mainly thanks to reduced pumping losses and in-cylinder heat transfer. NO and $\mathrm{CO}$ emissions are also improved under hydrogen injection at the considered lean burn limit 
$(\lambda=2)$ with respect to the stoichiometric operation. In particular, NO emissions can be almost removed at $5 \%$ hydrogen-enriched combustion and at the maximum investigated lean burn limit $(\lambda=2)$.

The adopted numerical methodology shows the capability to predict the effects of hydrogen-boosted combustion, opening the possibility to explore both performance and emission improvements in the whole engine operating map.

\section{Engine description, modeling and validation}

The tested engine is a twin-cylinder Spark-Ignition engine, designed according to the "downsizing" concept. It is equipped with a port fuel injector for each cylinder to supply the gasoline into the intake runner just upstream the intake valves. Engine is coupled to a waste-gated turbocharger. An external Low-pressure cooled EGR circuit is also mounted on the engine. Main features and performance of the examined engine are listed in the following Table 1. Experimental activity on the engine is carried out at low/medium loads and for various speeds. During tests, different performance variables are acquired, including air and fuel flows, brake torque/power and pollutant emissions. Each cylinder is also equipped with a pressure transducer, located between intake and exhaust valves, to measure a sequence of 270 consecutive in-cylinder pressure cycles. The acquired signals are then analyzed by a combustion analysis platform to derive the main indicated and combustion parameters. A reduced set of the overall acquired data is here studied in detail to prove the reliability of the proposed modeling approach. Table 2 shows the considered set of experimental operating points which is analyzed in terms of engine model validation, as discussed in the following.

Table 1. Main engine characteristics and performance.

\begin{tabular}{|c|c|}
\hline Engine model & $\begin{array}{c}\text { 2 cylinders, 8 valves, } \\
\text { PFI, Turbocharged }\end{array}$ \\
\hline Displacement & $875 \mathrm{~cm}^{3}$ \\
\hline Stroke/Bore & $86 \mathrm{~mm} / 80.5 \mathrm{~mm}$ \\
\hline Connecting rod length & $136.85 \mathrm{~mm}$ \\
\hline Compression ratio & 9.9 \\
\hline Max Brake Power & $64.6 \mathrm{~kW} @ 5500 \mathrm{rpm}$ \\
\hline Max Brake Torque & $146.1 \mathrm{Nm} @ 2500 \mathrm{rpm}$ \\
\hline
\end{tabular}

Table 2. Tested operating points.

\begin{tabular}{|c|c|c|c|c|c|}
\hline Case Label & IMEP, bar & Speed, rpm & $\boldsymbol{\lambda},-$ & EGR rate, $\%$ & $\mathbf{H}_{\mathbf{2}}$ vol., $\%$ \\
\hline 1800@8 & 8.0 & 1800 & 1.00 & 0.0 & 0.0 \\
\hline 2000@9 & 9.0 & 2000 & 1.00 & 0.0 & 0.0 \\
\hline 3000@7 & 7.0 & 3000 & 1.00 & 0.0 & 0.0 \\
\hline 3000@9 & 9.0 & 3000 & 1.00 & 5.0 & 0.0 \\
\hline 4000@5 & 5.0 & 4000 & 1.00 & 0.0 & 0.0 \\
\hline 4000@7 & 7.0 & 4000 & 1.00 & 0.0 & 0.0 \\
\hline 4000@9 & 9.0 & 4000 & 1.00 & 6.0 & 0.0 \\
\hline
\end{tabular}


A 1D engine model is developed in a commercial code, where a detailed engine schematization is provided by a network of $1 \mathrm{D}$ pipes, cylinders and relative junctions. Turbocharger operation is described by a standard map-based approach, while the flow permeability of the cylinder head is reproduced by the experimentally-derived steady flow coefficients. Engine model is also integrated with "in-house developed" sub-models to accurately describe the in-cylinder processes, including turbulence, combustion, heat transfer and pollutant emissions.

In particular, combustion is modeled by the fractal approach, developed in [20]. Combustion model is coupled to a turbulence sub-model [21], belonging to the K-k-T family. It describes the energy cascade mechanism from the mean flow kinetic energy, $\mathrm{K}$, to the turbulent one, $\mathrm{k}$, also taking into account a balance equation for the tumble angular momentum, T. Turbulence sub-model is properly tuned and a single set of tuning constants has been assigned at varying the engine speeds and the valve strategies [3]. Regarding the heat transfer modeling, a modified Hohenberg-like correlation is adopted, while the piston, cylinder liner and head temperatures are computed by the finite element approach, implemented into the $1 \mathrm{D}$ code. $\mathrm{NO}$ and $\mathrm{CO}$ emissions are computed through the implementation of 'users' sub-models in the $1 \mathrm{D}$ code $[13,22]$. In particular, CO concentrations are estimated by solving a simple chemical kinetic sub-model [23], while for NO concentration the semi-detailed chemical kinetic scheme proposed by Andrae [24] is solved in the burned gas zone.

Concerning the model validation, 1D model is set-up to adequately reproduce the available experimental findings. In particular, engine IMEP is regulated by a PID controller acting on the throttle (THR) valve opening, while the waste-gate (WG) valve is fully open. Gasoline injection is realized to replicate the experimental air/fuel (A/F) ratio. An additional PID controller is employed in the model to match the measured external EGR rate by controlling the EGR valve opening. The spark advance is automatically modified in the cycle-by-cycle simulation to realize the experimental combustion phasing $\left(\mathrm{MFB}_{50}\right)$. At low speed, a certain safety margin is also applied to the maximum boost pressure to avoid the surge occurrence [25]. The model accuracy is here proved against a limited set of measured points reported in Table 2, both in terms of global performance parameters, characteristic combustion durations and main exhaust emissions. Starting from the combustion process description, spark timing (Fig. 1a) and combustion core duration (Fig. 1b) are reproduced with good accuracy. Greater errors are found for the spark timing, because in these conditions the model fails in correctly estimating the early flame development. However, the above errors involve a slight impact on the prediction of the combustion phase, as demonstrated by the reduced average difference between computed and experimental combustion core durations. For the overall performance variables, Fig. 2 shows the numerical/experimental comparisons in terms of Indicated Specific Fuel Consumption (ISFC) (Fig. 2a) and of the In-cylinder pressure peak (Fig. 2b). ISFC shows an acceptable average absolute error (about 4.2\%), depending on the concurrent effects of both flow, combustion and heat transfer modeling. A nice correlation with the experimental findings is also obtained for the in-cylinder pressure peak, presenting an average absolute error of about $3.6 \%$.

Referring to the exhaust emissions, Fig. 3 reports the agreements between the 1D outcomes and the experimental findings for $\mathrm{NO}$ and $\mathrm{CO}$, respectively. The measured NO and $\mathrm{CO}$ species are predicted by the model with satisfactory accuracy (max percent error of about $10 \%$ ). In a previous authors' work [22], it was also demonstrated that NO and CO trends were adequately reproduced at varying the engine operating conditions. As a final remark, it is the case to underline that the adopted 1D model was extensively validated in [26] both in terms of combustion evolution and global performance, covering the entire engine operating domain. 


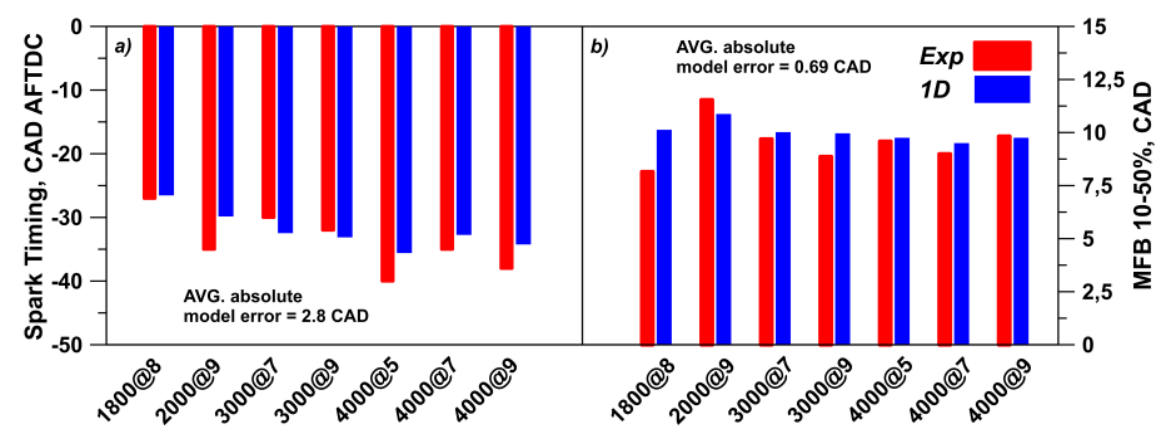

Fig. 1. Experimental vs Numerical comparison of Spark timing (a) and combustion core duration MFB $_{10-50 \%}(b)$.

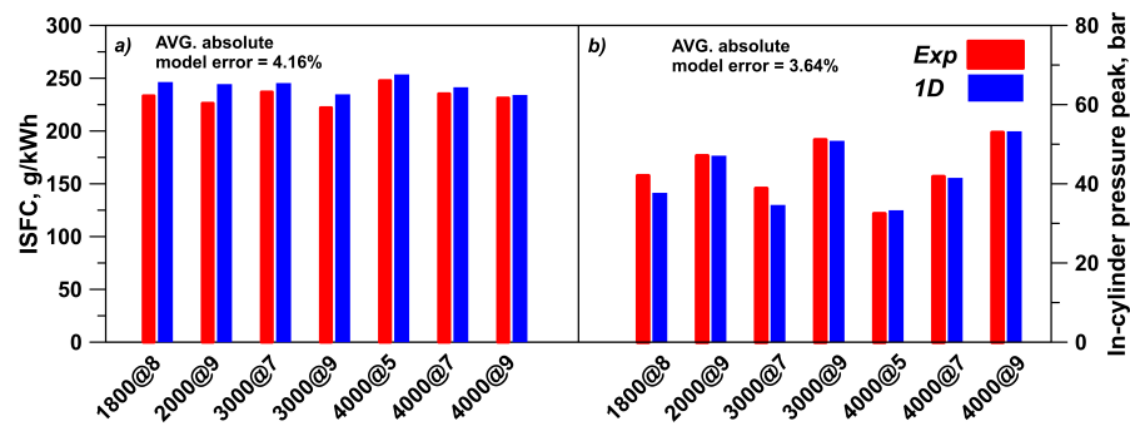

Fig. 2. Experimental vs Numerical comparison of ISFC (a) and In-cylinder pressure peak (b).

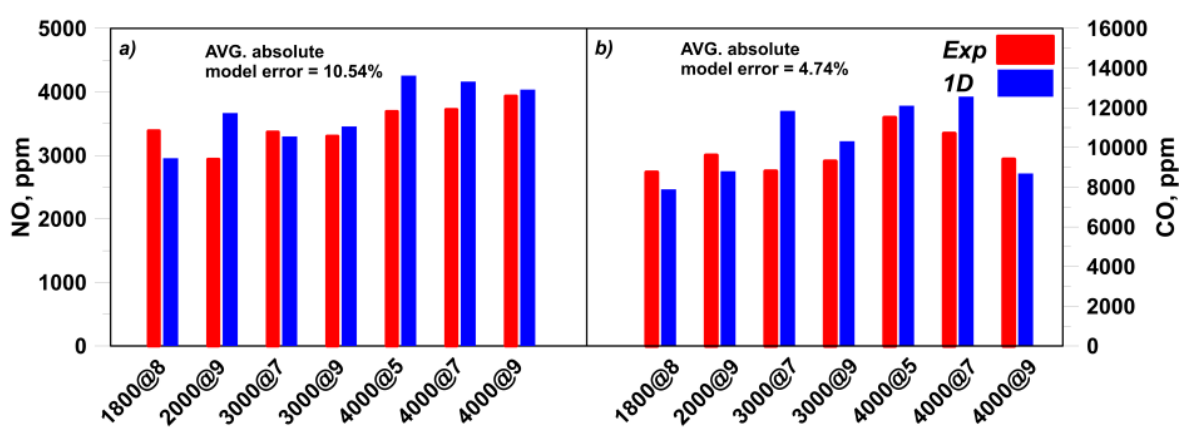

Fig. 3. Experimental vs Numerical comparison of $\mathrm{NO}$ (a) and CO (b) emissions.

\section{1D Simulations under hydrogen injection at low/medium loads: description and model set-up}

Once validated, 1D engine model is utilized to perform a numerical analysis at various loads and relative air/fuel ratio $(\lambda)$, exploring the effects of hydrogen injection in the intake manifold on both engine performance, combustion evolution and pollutant emissions. To this aim, 1D model is modified by virtually installing a low-pressure hydrogen injector in each intake runner, while the impact of gasoline/hydrogen blend on combustion process is taken into account through a dedicated laminar flame speed (LFS) correlation. 
The hydrogen is supplied by port injectors at ambient temperature and with the same injection timing of the gasoline port injectors. The amount of injected hydrogen is controlled to realize a constant percent level equal to $5 \%$ of hydrogen by volume into the engine intake air. For hydrogen-gasoline/air mixtures, the variation in the combustion evolution is considered by a laminar flame speed correlation developed at Istitute Francais du Petrol (IFPen) [18], which is believed to be highly reliable for hydrogen volumetric percentage in the intake air up to $6 \%$.
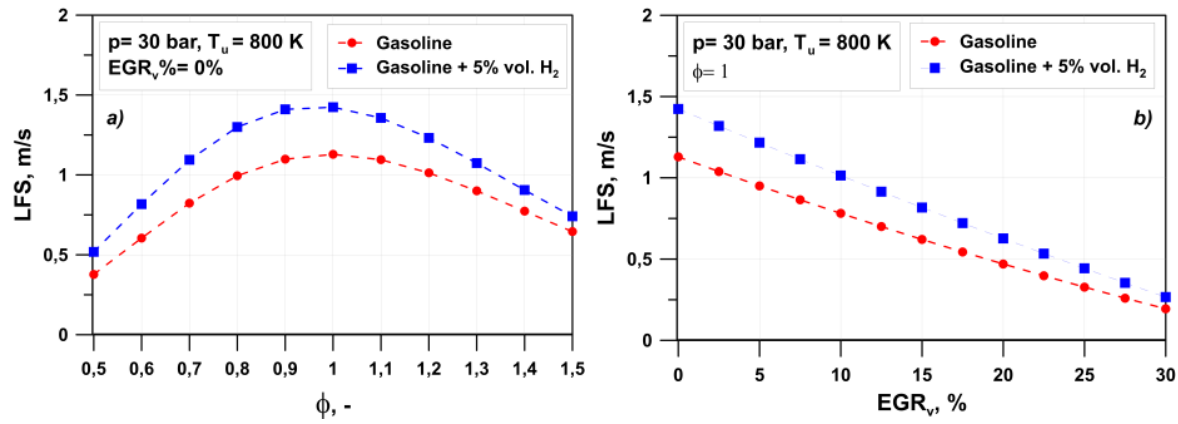

Fig. 4. Dependency of laminar flame speed correlation [18] on equivalence ratio (a) and on EGR volume fraction (b) for both gasoline and gasoline/hydrogen blend.

As clearly depicted in Fig. 4a-b, a higher LFS is obtained thanks to the hydrogen addition if compared to the sole gasoline. As expected, a maximum LFS is detected around $\Phi=1$ for the gasoline/ $\mathrm{H}_{2}$ blend (Fig. 4a) and the LFS decay at increasing the EGR is observed (Fig. 4b). In the light of the above discussed model modifications, two numerical investigations are carried out under hydrogen injection.

First, a sweep in the relative air/fuel ratio $(\lambda)$ is performed at fixed load point, i.e. 3 bar IMEP and $2250 \mathrm{rpm}$ (labelled as 2250@3). In this case, the benefits of the hydrogenboosted combustion can be explored up to the maximum acceptable lean mixture condition. The analysis is here limited up to $\lambda=2$, which is considered the maximum acceptable $\lambda$ level to limit the cycle-by-cycle variation for the analyzed engine [16].

A second analysis is realized at different knock-free load points and at constant speed of $2250 \mathrm{rpm}$ to highlight the advantages of the hydrogen-boosted combustion at lean burn limit $(\lambda=2)$ with respect to the stoichiometric operation of the engine fuelled with sole gasoline.

Referring to the model setup for the above discussed numerical applications, the experimental IMEP level is matched by a PID controller acting on the THR valve opening. If throttle valve is fully opened and the target load is not yet reached then a second PID controller will act on the WG valve opening to achieve the measured IMEP. The injected gasoline mass is controlled in order to realize a target $\lambda$ level, while the hydrogen injection is regulated to realize a constant value of $5 \%$ of hydrogen by volume in the engine intake air. Hydrogen injection is actuated only in the case of lean combustion to support the flame development and the combustion stability. The spark advance is automatically modified in the cycle-by-cycle simulation to realize the operation at Maximum Brake Torque (MBT) condition $\left(\mathrm{MFB}_{50}=8 \mathrm{CAD}\right.$ AFTDC).

\section{Results discussion}

Numerical outcomes show that hydrogen injection for the examined SI engine involves efficiency improvements for all the considered operating conditions. As already pointed 
out, the numerical results under hydrogen injection are obtained only for the lean mixture conditions. Indeed, the sweep in the relative air/fuel ratio $(\lambda)$ is realized starting from the value of $\lambda=1.15$ for the gasoline $/ \mathrm{H}_{2}$ case. The reference engine operation corresponding to the stoichiometric gasoline/air mixture is named "BASE" solution in the following figures.

Referring to the parametric analysis at varying $\lambda$ level for the operating point namely $2250 \mathrm{rpm} / 3$ bar IMEP (2250@3), Fig. 5a depicts the indicated efficiency trend for both gasoline $/ \mathrm{H}_{2}$ and gasoline cases.

The adoption of the sole gasoline does not allow to extend the lean burn limit beyond $\lambda=1.25$, where the indicated efficiency benefit is equal to $6.7 \%$. The limit of $\lambda=1.25$ is established in order to avoid an unacceptable increase in the cycle-by-cycle variation for the examined engine. This solution has to be considered as a purely theoretical case. The discussed drawback of gasoline case is overcome by the hydrogen injection $\left(5 \%\right.$ of $\mathrm{H}_{2}$ by volume in the intake air), reaching an indicated efficiency benefit of $18.8 \%$ at $\lambda=2$. This advantage is realized mainly thanks to the decreased pumping losses (Fig. 6a) and the incylinder heat losses (Fig. 6b). Referring to the combustion evolution, a slight increase in combustion core duration (MFB $10-50 \%$ ) is observed for gasoline $/ \mathrm{H}_{2}$ case at $\lambda=2$ compared to gasoline-stoichiometric condition (Fig. 5b), due to the opposite effects of an increased LFS with hydrogen addition and its reduction due to the air/gasoline mixture leaning. The early stage of combustion (MFB $0-10 \%$ ) against $\lambda$ for hydrogen/gasoline case in Fig. $5 \mathrm{~b}$ shows an increasing trend. The maximum increase of MFB $0-10 \%$ under hydrogen injection is realized at $\lambda=2$, where a level substantially comparable to the one achieved by the sole gasoline at $\lambda=1.25$ is observed.
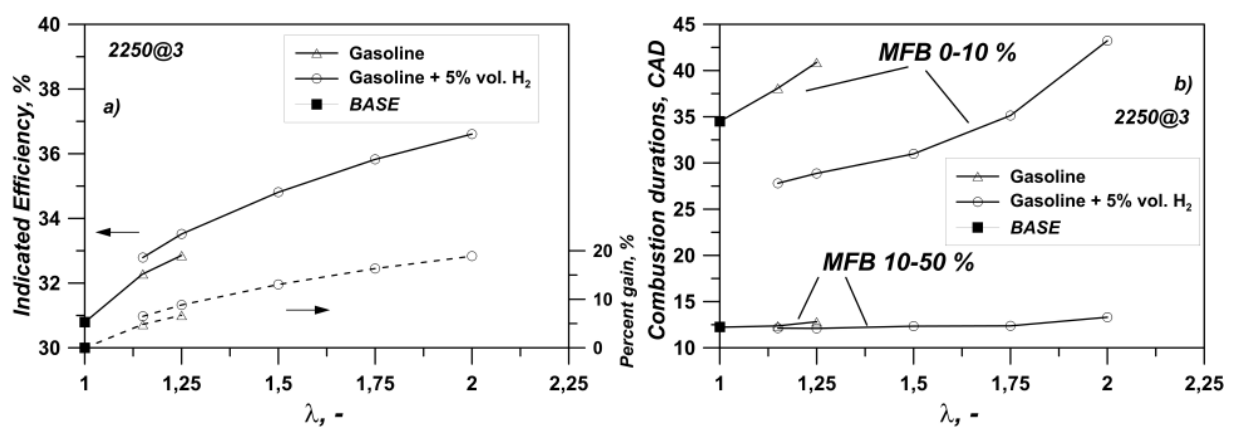

Fig. 5. Indicated Efficiency (a) and characteristic combustion durations (b) vs $\lambda$ at $2250 @ 3$.
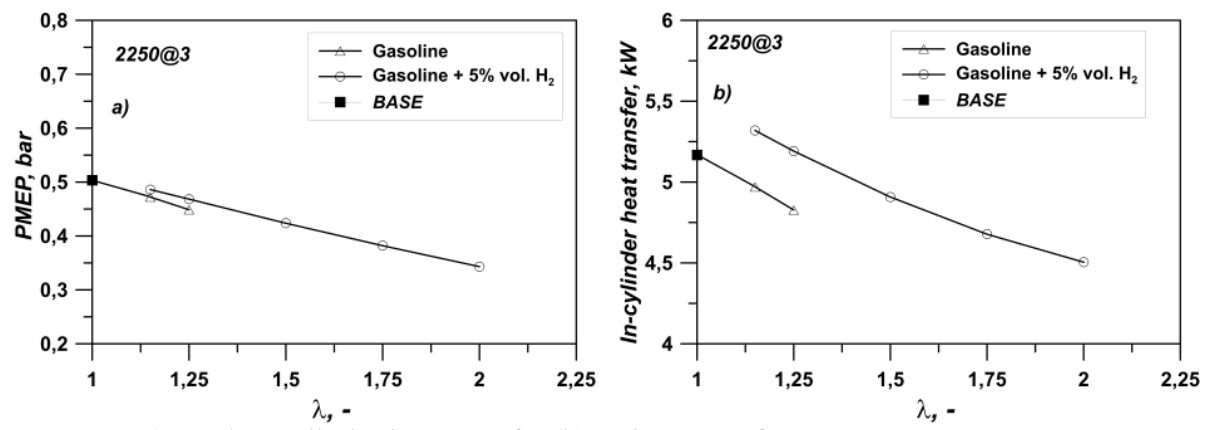

Fig. 6. PMEP (a) and In-cylinder heat transfer (b) vs $\lambda$ at 2250@3.

As expected, an increasing trend with $\lambda$ of the air flow rate is obtained (Fig. 7a) to recover the engine torque under ultra-lean mixture conditions. The temperature at the 
engine exhaust in Fig. $7 \mathrm{~b}$ shows a decreasing trend with $\lambda$, allowing for the mitigation of turbine thermal stress.

To deepen the understanding of hydrogen effects at considered knock-free operating point, the in-cylinder pressure and temperature cycles for BASE case (gasoline at $\lambda=1$ ) and for gasoline $/ \mathrm{H}_{2}$ case at $\lambda=2$ are depicted in Fig. 8a-b. Looking at Fig. 8a, the increased boost level caused by $\mathrm{H}_{2}$ addition determines a higher pressure at TDC, while the slower burning rate at $\lambda=2$ limits the increase of the pressure peak. The air/gasoline mixture dilution helps to realize a significant reduction in the burned zone temperature $\left(T_{b}\right)$. Indeed, a clear $T_{b}$ decrease is obtained for the case of $\mathrm{H}_{2}$ addition and $\lambda=2$ with respect to the BASE case (Fig. 8b). The same Figure shows that the spark timing is advanced, thus confirming the above discussed combustion lengthening.
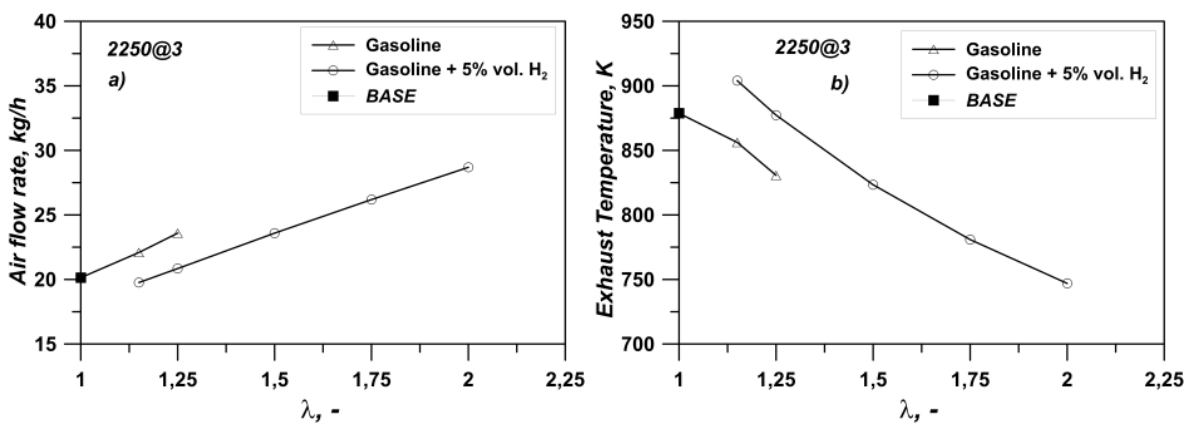

Fig. 7. Air flow rate (a) and Engine Exhaust Temperature (b) vs $\lambda$ at 2250@3.
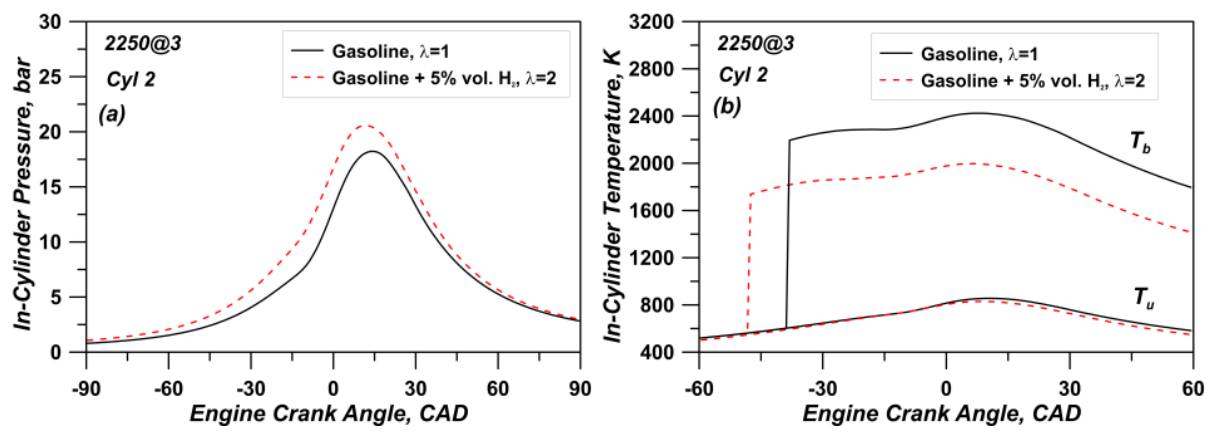

Fig. 8. In-Cylinder pressure (a) and Burned/Unburned Temperature evolutions (b) at 2250@3 for Cyl 2: comparison of stoichiometric gasoline case and $\lambda=2-\mathrm{H}_{2} /$ gasoline case.

$\mathrm{NO}$ and $\mathrm{CO}$ emission trends are plotted in Fig. 9a-b. In particular, referring to the gasoline/ $\mathrm{H}_{2}$ case, Fig. 9a shows an initial increase in the NO emission followed by a considerable reduction up to a very low level $(23.7 \mathrm{ppm}$ at $\lambda=2)$. The initial NO increase is probably due to the combination of a greater in-cylinder oxygen concentration and of a substantially unchanged burned zone temperature. At $\lambda=2$, the marked reduction in the burned zone temperature is the prevailing driver for the almost null NO production.

As a further consideration, the employment of sole gasoline at the corresponding lean burn limit $(\lambda=1.25)$ does not show the capability to eliminate the NO emission: about 560 ppm are still realized at $\lambda=1.25$ in Fig. 9a. Referring to the $\mathrm{CO}$ emission, it is drastically reduced with the $\lambda$ increase (Fig. 9b). This trend is experimentally confirmed in the literature and the hydrogen addition has not obvious effects on the $\mathrm{CO}$ emission trend [27]. However, very low CO values are predicted by the adopted sub-model (5-10 ppm under 
lean combustion). Indeed, $\mathrm{CO}$ sub-model underestimates the $\mathrm{CO}$ levels under lean combustion, because it does not include the $\mathrm{CO}$ generated by the low-temperature oxidation of the Unburned HC [28]. In addition, close to the lean burn limit higher amount of CO may be generated $[29,30]$.
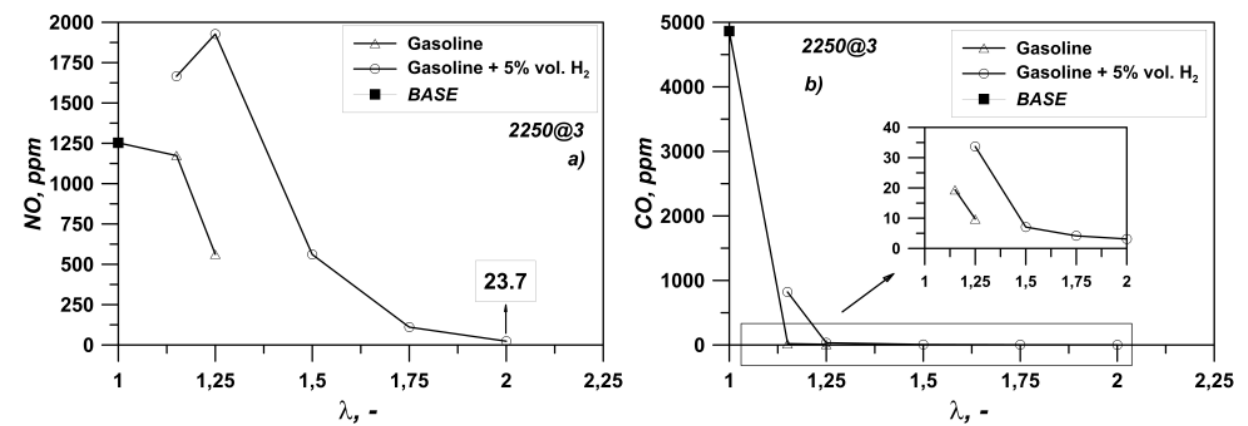

Fig. 9. NO ppm (a) and CO ppm (b) emissions at engine exhaust vs $\lambda$ at 2250@3.

As said, the second application consists in a load sweep under knock-free operation to compare the advantages of hydrogen-related lean combustion with the gasoline stoichiometric operation of the engine. The same engine speed and hydrogen amount of the previous analysis are here considered, while $\lambda$ level under hydrogen injection is fixed at 2 to guarantee a certain safety margin with respect to the stability of combustion and torque delivery.

Fig. 10a shows that hydrogen addition allows improved indicated efficiency for all IMEP levels, even if this is achieved at the expense of a higher in-cylinder pressure peak (Fig. 10b). Consequently, a greater mechanical stress for the engine is realized, while the thermal stress is limited mainly thanks to the LTC (Fig. 8b).
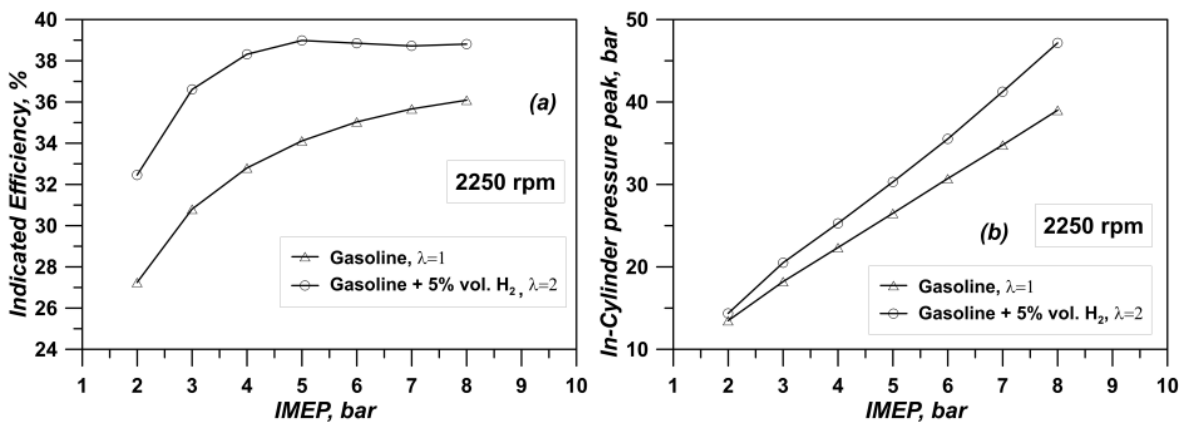

Fig. 10. Indicated Efficiency (a) and In-cylinder pressure peak (b) vs IMEP at $2250 \mathrm{rpm}$.

Fig. 11a depicts the percent advantage of Indicated Efficiency, ranging from the lowest value of about $7.5 \%$ at 8 bar IMEP up to the maximum value of $19.1 \%$ at 2 bar IMEP. Indicated efficiency benefits arise from the combination of both reduced pumping losses (Fig. 11b) and reduced in-cylinder heat losses (Fig. 11c). Indeed, Fig. 11b-c report the absolute gains in terms of PMEP (bar) and in-cylinder heat transfer $(\mathrm{kW})$, respectively. They are computed as difference between hydrogen injection and pure gasoline cases. Minor indicated efficiency gains are obtained at the higher loads. Indeed, at increasing the load a higher boost pressure is required and a partial WG closing is needed for loads above 6 bar IMEP, resulting in fewer advantages of pumping losses (Fig. 11b). On the other hand, the in-cylinder heat transfer benefits exert a greater impact on the engine efficiency at 
decreasing the load. NO and CO emissions are remarkably reduced with hydrogen injection and $\lambda=2$, as represented in Fig. 12a-b, respectively. Under hydrogen injection, a slight increase in NO emission as the IMEP increases is observed (Fig. 12a), due to the higher load-induced burned zone temperature. Fig. 12b shows an almost null $\mathrm{CO}$ emission for hydrogen injection case. Actually, CO levels under hydrogen-boosted ultra-lean combustion are still quite low but they are not close to null level. As already said, the adopted $\mathrm{CO}$ sub-model does not take into account the $\mathrm{CO}$ species generated by the lowtemperature oxidation of Unburned HC [28]. Therefore, the results presented in Fig. 12b has to be interpreted as a noticeable $\mathrm{CO}$ emission reduction instead of an accurate quantitative prediction for the lean combustion supported by hydrogen addition.

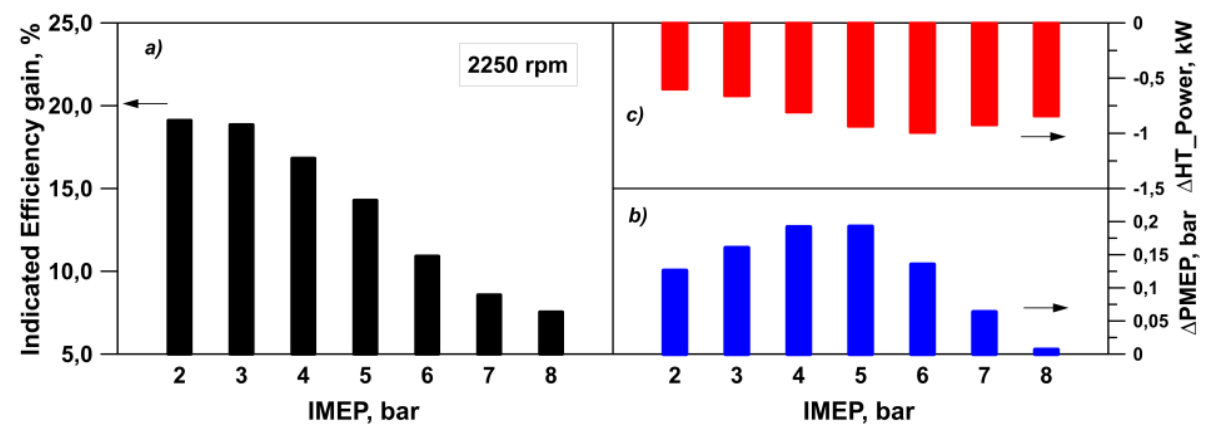

Fig. 11. Indicated Efficiency gain $\%$ (a), $\triangle \mathrm{PMEP}(\mathrm{b})$, and $\triangle \mathrm{HT}$-Power (c) vs IMEP at $2250 \mathrm{rpm}$.
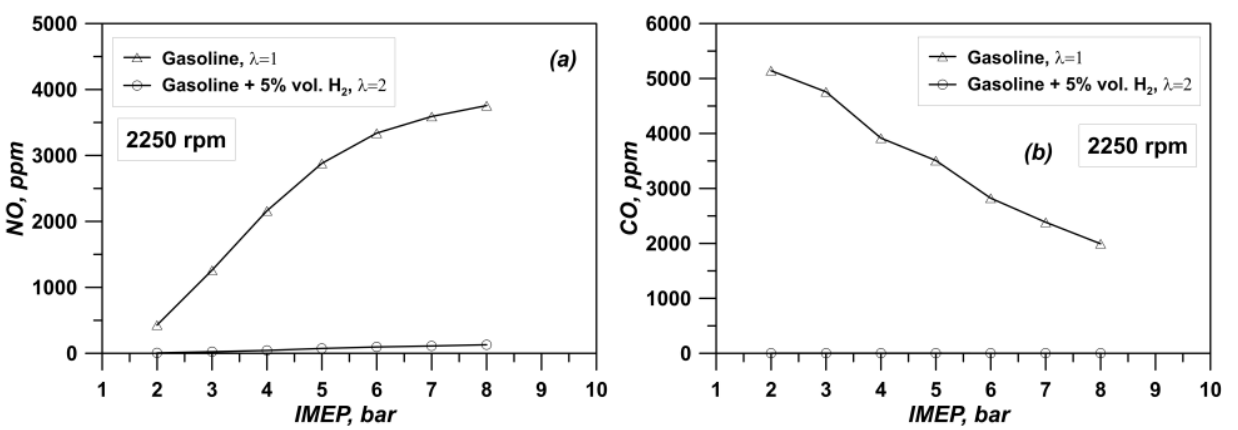

Fig. 12. NO ppm (a) and CO ppm (b) vs IMEP at $2250 \mathrm{rpm}$.

\section{Conclusions}

In this work, the impact of hydrogen injection on performance and emissions of a twincylinder gasoline spark ignition engine has been analyzed by a $1 \mathrm{D}$ model. In a preliminary phase, the base engine has been experimentally tested at various speeds and loads, collecting the main performance variables and exhaust emissions, as well.

A 1D model of the whole engine has been realized within a commercial code and integrated with refined in-cylinder sub-models, including turbulence, combustion, heat transfer and emissions. The engine model has been validated against the available experimental findings, denoting a satisfactory agreement both in terms of performance, combustion evolution and emissions. The validated 1D model has been utilized in a predictive manner for an application study consisting in the hydrogen injection within the intake runners. The numerical investigations have been oriented to a reduced set of operating points, characterized by constant speed of $2250 \mathrm{rpm}$ and knock-free loads. Only $5 \%$ of hydrogen by volume in the intake air has been considered in the application study. 
A parametric analysis in the relative air/gasoline ratio $(\lambda)$ has been performed considering a fixed speed/load point, i.e. $2250 \mathrm{rpm}$ and 3 bar IMEP. The maximum $\lambda$ level has been limited up to 2 , in order to avoid an excessive cycle-by-cycle variation for the tested engine and the employed amount of hydrogen. Relevant engine efficiency benefits (up to about 19\%) have been obtained in lean combustion conditions, mainly thanks to the reduction in the pumping losses and to the lowering of the in-cylinder heat losses. Low temperature combustion has been realized, contributing to the almost complete elimination of NO emissions, while the availability of a greater oxygen concentration promotes the oxidation reactions and the drastically reduction of the $\mathrm{CO}$ levels.

The analysis in a load sweep at $2250 \mathrm{rpm}$ has pointed out that very high efficiency benefits (ranging from $7.5 \%$ up to $19 \%$ ) can be achieved, thanks to the hydrogen injection under ultra/lean mixture conditions. NO and CO emissions have been drastically reduced if compared to the stoichiometric air/gasoline mixture operations. The hydrogen-related ultralean combustion also involves a reduction in the Unburned HC emissions, as demonstrated in the literature, but this solution still poses the need for an oxidizing catalyst at the engine exhaust for the effective $\mathrm{HC}$ abatement. In a future activity development, the discussed application of hydrogen injection in the intake manifold will be verified through dedicated experiments, also including different hydrogen volumetric fractions, wider engine operating conditions and the $\mathrm{H}_{2}$ influence on the Unburned $\mathrm{HC}$ emissions. In this way, the predictivity of the 1D modeling approach will be further enhanced, and the simulations could be extended to identify the optimal hydrogen injection strategy and to cover the effects of hydrogen injection in the entire engine operating domain.

\section{References}

1. European Environment Agency, Annual European Union Greenhouse Gas Inventory 1990-2012 and Inventory Report, Report No.: 9 (2014).

2. G. Police, S. Diana, V. Giglio, et al. Downsizing of SI engines by turbo-charging. Proc. of ASME Conf. on Eng. Systems Design and Analysis, Vol 42517, pp. 463-476, (2006). https://doi.org/10.1115/ESDA2006-95215.

3. L. Teodosio, D. Pirrello, F. Berni, V. De Bellis et al. Impact of intake valve strategies on fuel consumption and knock tendency of a spark ignition engine. Applied Energy 216, 91-104, (2018). https://doi.org/10.1016/j.apenergy.2018.02.032.

4. L. Teodosio, V. De Bellis, F. Bozza, D. Tufano. Numerical Study of the Potential of a Variable Compression ratio Concept applied to a downsized turbocharged VVA Spark Ignition engine. SAE Technical Paper 2017-24-0015, (2017). https://doi.org/10.4271/2017-24-0015.

5. M. Cordier, O. Laget, F. Duffour, X. Gautrot, et al. Increasing Modern Spark Ignition Engine Efficiency: A comprehension study of High CR and Atkinson cycle. SAE Technical Paper 2016-01-2172, (2016). https://doi.org/10.4271/2016-01-2172.

6. F. Bozza, L. Teodosio, V. De Bellis, D. Cacciatore, et al. A Modelling study to analyse the compression ratio effects on combustion and knock phenomena in a highperformance spark-ignition GDI engine. Int. Review on Modelling and Simulations 11 (3): 187-197, (2018). https://doi.org/10.15866/iremos.v11i3.13771.

7. E. Galloni, G. Fontana, and R. Palmaccio. Numerical Analyses of EGR techniques in a turbocharged spark-ignition engine. Appl. Therm. Eng. 39, 95-104, (2012). https://doi.org/10.1016/j.applthermaleng.2012.01.040.

8. C. Tornatore, F. Bozza, V. De Bellis, L. Teodosio et al. Experimental and numerical study on the influence of cooled EGR on knock tendency, performance and emissions of a downsized spark-ignition engine. Energy 172: 968-976, (2019). https://doi.org/10.1016/j.energy.2019.02.031. 
9. A. D’Adamo, F. Berni, S. Breda, M. Lugli et al. A numerical investigation on the potentials of water injection as a fuel efficiency enhancer in highly downsized GDI engines. SAE Technical Paper 2015-01-0393, (2015). https://doi.org/10.4271/2015-010393.

10. A. Boretti. Water Injection in directly injected turbocharged spark ignition engine. Applied Thermal Engineering $52 \quad$ (1): 62-68, (2013). https://doi.org/10.1016/j.applthermaleng.2012.11.016.

11. Z. Ran, D. Hariharan, B. Lawler, S. Mamalis. Exploring the potential of ethanol, CNG, and syngas as fuels for lean spark-ignition combustion - an experimental study. Energy 191, (2020). https://doi.org/10.1016/j.energy.2019.116520.

12. X. Wu, R. Daniel, G. Tian, H. Xu et al. Dual-injection: The flexible, bi-fuel concept for spark ignition engines fueled with various gasoline and bio-fuel blends. Applied Energy 88 (7): 2305-14, (2011). https://doi.org/10.1016/j.apenergy.2011.01.025.

13. F. Bozza, D. Tufano, E. Malfi, L. Teodosio et al. Performance and Emissions of an advanced multi-cylinder SI engine operating in ultra-lean conditions. SAE Technical Paper 2019-24-0075, (2019). https://doi.org/10.4271/2019-24-0075.

14. L. Teodosio, V. De Bellis, F. Bozza. Combined effects of valve strategies, compression ratio, water injection, and cooled EGR on the Fuel Consumption of a Small Turbocharged VVA Spark-Ignition Engine. SAE Int. Journal of Engines 11 (6): 643656, (2018). https://doi.org/10.4271/2018-01-0854.

15. F. Bozza, V. De Bellis, L. Teodosio, D. Tufano et al. Techniques for $\mathrm{CO}_{2}$ emission reduction over a WLTC. A numerical comparison of increased compression ratio, cooled EGR and water injection. SAE Technical Paper 2018-37-0008, (2018). https://doi.org/10.4271/2018-37-0008.

16. C. Ji, S. Wang. Effect of hydrogen addition on combustion and emissions performance of a spark ignition gasoline engine at lean conditions. Int. J. Hydrogen Energy 34 (18): 7823-34, (2009). https://doi.org/10.1016/j.ijhydene.2009.06.082.

17. T. D'Andrea, P.F. Henshaw, D.K. Ting. The addition of hydrogen to a gasoline-fuelled SI engine. Int. J. Hydrogen Energy 29 (14): 1541-52, (2004). https://doi.org/10.1016/j.ijhydene.2004.02.002.

18. N. Iafrate, M. Matrat, J. M. Zaccardi. Numerical investigations on hydrogen-enhanced combustion in ultra-lean gasoline spark-ignition engines. Int. J. Engine Research 1-15, (2019). https://doi.org/10.1177/1468087419870688.

19. T. Tahtough, F. Halter, E. Samson, C. Mounmaim-Rousselle (2011). Effects of hydrogen addition under lean and diluited conditions on combustion characteristics and emissions in a spark ignition engine. Int. J. Eng. Research 12 (5): 466-483. https://doi.org/10.1177/1468087411409309.

20. F. Bozza, A. Gimelli, S.S. Merola and B.M. Vaglieco. Validation of a Fractal Combustion through flame imaging. SAE Transaction 114 (3): 973-987, (2005). doi: $10.2307 / 44722057$.

21. F. Bozza, L. Teodosio, V. De Bellis, S. Fontanesi et al. A Refined 0D Turbulence Model to predict Tumble and Turbulence in SI Engines. SAE Int. J. Engines 12: 15-30, (2019). doi: 10.4271/03-12-01-0002.

22. L. Marchitto, L. Teodosio, C. Tornatore, G. Valentino et al. Experimental and 1D Numerical Investigations on the Exhaust emissions of a Small Spark Ignition Engine considering the cylinder-by-cylinder variability. SAE Technical Paper 2020-01-0578, (2020). https://doi.org/10.4271/2020-01-0578.

23. T. Cerri, G. D'Errico, G. Montenegro, A. Onorati et al. A Novel 1D Co-Simulation Framework for the prediction of Tailpipe Emissions under different IC engine operating conditions. SAE Technical Paper 2019-24-0147, (2019). https://doi.org/10.4271/2019-24-0147. 
24. J. Andrae. Comprehensive chemical kinetic modeling of toluene reference fuels oxidation. Fuel 107: 740-748, (2013). https://doi.org/10.1016/j.fuel.2013.01.070.

25. F. Bozza, V. De Bellis, L. Teodosio and A. Gimelli. Numerical analysis of the transient operation of a turbocharged diesel engine including the compressor surge. Proc. of the Inst. of Mech. Eng., Part D. J. Aut. Eng. 227: 1503-17, (2013). https://doi.org/10.1177/0954407013501668.

26. L. Teodosio, F. Bozza, D. Tufano, P. Giannattasio et al. Impact of the laminar flame speed correlation on the results of a quasi-dimensional combustion model for Sparkignition engine. Energy Procedia 148: 631-638, (2018). https://doi.org/10.1016/j.egypro.2018.08.151.

27. R. Niu, X. Yu, Y. Du, H. Xie et al. Effect of hydrogen proportion on lean burn performance of a dual fuel SI engine using hydrogen direct-injection. Fuel 186: 792799, (2016). https://doi.org/10.1016/j.fuel.2016.09.021.

28. Technical Reports of Eagle project on the Pre-chamber SI engines, (2019). https://h2020-eagle.eu/Index.aspx.

29. A. K. Agarwal, A. P. Singh, R. K. Maurya. Evolution, challenges and path forward for low temperature combustion engines. Progress in Energy and Combustion Science 61: 1-56, (2017). https://doi.org/10.1016/j.pecs.2017.02.001.

30. M. Christensen, B. Johansson, P. Einewall. Homogeneous charge compression ignition (HCCI) using isooctane, ethanol and natural gas - A comparison with spark ignition operation. SAE Technical Paper 972874, (1997). https://doi.org/10.4271/972874. 